Pacific Journal of Mathematics

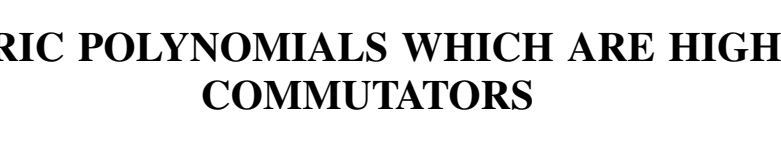




\title{
MATRIC POLYNOMIALS WHICH ARE HIGHER COMMUTATORS
}

\author{
EDMOND D. Dixon
}

Let $A$ be an $n \times n$ matrix defined over a field $F$ of characteristic greater than $n$. For each $n \times n$ matrix $X$ we define

$$
\begin{aligned}
X_{1} & =[A, X]_{0}=X \\
X_{h+1}=[A, X]_{h} & =\left[A, X_{h}\right]=A X_{h}-X_{h} A
\end{aligned}
$$

for each positive integer $h$. Then $X$ is defined to be $k$-commutative with $A$ if and only if

$$
[A, X]_{k}=0, \quad[A, X]_{k-1} \neq 0 .
$$

Let $P(x)$ be a polynomial such that $P(A) \neq 0$. Specifically, assume that

$$
P(A)=\sum_{i=p}^{n-1} \lambda_{i} A^{i} \neq 0
$$

where $p$ is a positive integer, each $\lambda_{i}$ is a scalar from $F$, and $\lambda_{p} \neq 0$. In this paper we study, for each positive integer $k$, the matrices $X$ such that

$$
[A, X]_{k}=P(A) .
$$

We specify a polynomial $P(A)$ in the form (3) and show how the maximal value of $k$ for which (4) has a solution depends on the polynomial $P(A)$. In Theorem 3 it is assumed that $A$ is nonderogatory. Since the only matrices which commute with $A$ in this case are polynomials in $A$, we are, in effect, establishing a more precise bound for $k$ in (2) by predetermining $X_{k}$.

In the derogatory case, a matrix which is not a polynomial in $A$ may commute with $A$. However, Theorem 4 shows that if we choose a polynomial $P(A)$ as $X_{k}$, then the maximal value of $k$ depends on the polynomial $P$.

The problem of determining the maximal value of $k$ for which (2) has a solution has been studied by Roth [8] and others. Roth's results are stated in terms of the maximal degrees of the elementary divisors of the matrix $A$. In particular, he showed that there exists a matrix $X$ satisfying (2) for some $A$ if $k \leqq 2 n-1$.

Nilpotent case. Throughout the paper we assume that $A$ is in Jordan canonical form, since $[a, X]_{k}=P(A)$ if and only if

$$
\left[B A B^{-1}, B X B^{-1}\right]_{k}=B P(A) B^{-1} \text {. }
$$


The following notation introduced by W. V. Parker is used to simplify the proofs of the theorems.

Definition. Let $M_{s}$ for any integer $s$ such that $-n+1 \leqq s \leqq$ $m-1$ be the set of all $n \times m$ matrices in which all elements are zero except those for which $j-i=s$ ( $i$ denotes the row and $j$ denotes the column in which the element appears). If $s>m-1, M_{s}$ is defined to be the set consisting of only the zero matrix. A particular member of $M_{s}$ will be denoted by $D_{s}$ and will be called an $s$-stripe matrix. Note that if $X$ is any $n \times m$ matrix then $X$ can be written uniquely as $X=\sum_{s=-n+1}^{m-1} D_{s}$ where $D_{s}$ is an element of $M_{s}$.

If $A_{1}$ and $A_{2}$ are $n \times n$ and $m \times m$ nilpotent nonderogatory matrices in Jordan canonical form and if $D_{s}=\left(d_{i j}\right)$ is an $n \times m$ element of $M_{s}$ where $s$ is any integer such that $-n+1 \leqq s \leqq m-1$, let $f\left(D_{s}\right)=A_{1} D_{s}-D_{s} A_{2}$ and $f^{k}\left(D_{s}\right)=A_{1} f^{k-1}\left(D_{s}\right)-f^{k-1}\left(D_{s}\right) A_{2}$. It is easily seen that $f^{k}\left(D_{s}\right)$ is an element of $M_{s+k}$. Notice that the element in the $i j$ position of $f\left(D_{s}\right)$, where $j-i=s+1$, is $d_{i+1, j}-d_{i, j-1}$ for $i \neq 1$. The element in the $n j$ position is $-d_{n, j-1}$ if $j \neq 1$; the element in the $i 1$ position is $d_{i+1,1}$ if $i \neq n$; and the element in the $n 1$ position is zero.

Lemma 1. If $A$ is an $n \times n$ nilpotent nonderogatory matrix in Jordan canonical form, if $X$ is an $n \times n$ matrix, and if

$$
M=[A, X]=A X-X A,
$$

then the trace of $M$ is zero and the trace of every subdiagonal stripe of $M$ is zero.

Proof. Any $n \times n$ matrix $X$ may be written as $\sum_{s=-n+1}^{n-1} D_{s}$ where $D_{s}$ is an element of $M_{s}$. Thus

$$
[A, X]=\left[A, \sum_{s=-n+1}^{n-1} D_{s}\right]=\sum_{s=-n+1}^{n-1}\left[A, D_{s}\right]
$$

If $s<0$, then $\left[A, D_{s}\right]$ is a matrix such that the sum of the nonzero elements is zero. The matrix $\left[A, D_{s}\right]$ forms the $(s+1)$-stripe of $M$. This completes the proof of the lemma.

If $A$ is an $n \times n$ nilpotent nonderogatory matrix in Jordan canonical form then for any positive integer $s<n,\left(A^{T}\right)^{s} A^{s}$ plays the part of a "lower identity" which we denote by $L_{s}$. That is,

$$
\left(A^{T}\right)^{s} A^{s}=\left(\begin{array}{ll}
0 & 0 \\
0 & I_{n-s}
\end{array}\right)=L_{s} .
$$


Similarly,

$$
A^{s}\left(A^{T}\right)^{s}=\left(\begin{array}{cc}
I_{n-s} & 0 \\
0 & 0
\end{array}\right)=U_{s}
$$

which we call an "upper identity".

Using the above, we prove the following lemma.

LEMMA 2. Let $A$ be an $n \times n$ nilpotent nonderogatory matrix in Jordan canonical form. Let $L_{s}$ and $U_{s}$ be as defined above. Then

$$
L_{s}(I-A) L_{s+k}=(I-A) L_{s+k}
$$

and

$$
U_{s+k}(I-A) U_{s}=U_{s+k}(I-A),
$$

where $k$ is any positive integer less than $n-s$.

Proof. If we partition $I-A$ as follows:

$$
(I-A)=\left(\begin{array}{cc}
M & 0 \\
* & N
\end{array}\right)
$$

where $M$ is $s \times(s+k)$, then

$$
L_{s}(I-A) L_{s+k}=\left(\begin{array}{cc}
0 & 0 \\
* & N
\end{array}\right) L_{s+k}=\left(\begin{array}{cc}
0 & 0 \\
0 & N
\end{array}\right)=(I-A) L_{s+k} .
$$

The proof of (8) is similar.

Let $V=(1,1, \cdots, 1)$, a $1 \times n$ vector, and let $V_{s}=V D_{s}$. That is, $V_{s}$ is the vector in which each element represents a column sum in $D_{s}$, and since the columns in $D_{s}$ have at most one nonzero element, $V_{s}$ simply displays these elements in the form of a row vector. To simplify the notation we will let $V_{s+k}=V D_{s+k}$ where $D_{s+k}=\left[A, D_{s}\right]_{k}$ for some matrix $D_{s}$. In other words, the added subscript, $k$, implies that $V_{s+k}$ is the result of $k$ commutations. From now on, $s$ will denote a nonnegative integer, $0 \leqq s \leqq n-1$, and subdiagonal stripes of $X$ will be denoted by $D_{-s}$. Also, the nontrivial subvector in $V_{s}$ will be denoted by $w_{n-s}$, and the nontrivial subvector in $V_{s}$ will be denoted by $\hat{w}_{n-s}$. Thus

$$
V_{s}=\left(0,0, \cdots, 0, d_{1, s+1}, d_{2, s+2}, \cdots, d_{n-s, n}\right)=\left(0_{s}, w_{n-s}\right) .
$$

Similarly,

$$
V_{-s}=\left(d_{s+1,1}, d_{s+2,2}, \cdots, d_{n, n-s}, 0, \cdots, 0\right)=\left(\hat{w}_{n-s}, 0_{s}\right) .
$$

The following lemma is a vital part of the proof of Theorem 1 . 
Lemma 3. If $k$ is a positive integer and if $V_{s}, A, U_{s}$, and $L_{s}$ are as defined above, then

(i) $V_{s+k}=V_{s}(I-A)^{k} L_{k}$,

(ii) $V_{-s+k}=V_{-s} U_{s}(I-A)^{k}$ if $k \leqq s$,

(iii) $V_{-s+k}=V_{-s} U_{s}(I-A)^{k} L_{k-s}$ if $k>s$.

Proof. Case (i). If $k=1$, from (7) and (9)

$$
V_{s}(I-A) L_{s+1}=\left(0_{s}, w_{n-s}\right)\left(\begin{array}{cc}
0 & 0 \\
0 & N
\end{array}\right) \text {. }
$$

In this case $N$ has dimensions $(n-s) \times(n-s-1)$, so $N$ has $(-1)$ 's on the diagonal and 1's on the first subdiagonal. But

$$
\left(0_{s}, w_{n-s}\right)\left(\begin{array}{cc}
0 & 0 \\
0 & N
\end{array}\right)=\left(0_{s}, w_{n-s}\right) N=\left(0_{s+1}, w_{n-s-1}\right)
$$

where $w_{n-s-1}$ has only $n-s-1$ elements of the form $\left(d_{i+1, s+i+1}-d_{i, s+i}\right)$, and this is $V_{s+1}$. Therefore

$$
V_{s+1}=V_{s}(I-A) L_{s+1} .
$$

Similarly,

$$
V_{s+2}=V_{s+1}(I-A) L_{s+2}=V_{s}(I-A) L_{s+1}(I-A) L_{s+2} .
$$

But by Lemma 2,

$$
L_{s+1}(I-A) L_{s+2}=(I-A) L_{s+2} .
$$

Thus $V_{s \div 2}=V_{s}(I-A)^{2} L_{s+2}$, and by induction it follows that

$$
V_{s \div k}=V_{s}(I-A)^{k} L_{s+k} .
$$

In particular,

$$
V_{0 \div \frac{1}{0}}=V_{0}(I-A)^{k} L_{k} .
$$

Case (ii). From (10),

$$
V_{-s} U_{s}(I-A)=V_{-s}\left(\begin{array}{cc}
I_{n-s} & 0 \\
0 & 0
\end{array}\right)\left(\begin{array}{cc}
M & 0 \\
* & N
\end{array}\right)=\left(\hat{w}_{n-s}, 0_{s}\right)\left(\begin{array}{cc}
M & 0 \\
0 & 0
\end{array}\right)
$$

where $M$ has dimensions $(n-s) \times(n-s+1)$ and so has 1 's on the diagonal and $(-1)$ 's on the first superdiagonal. But

$$
\left(\widehat{w}_{n-s+1}, 0_{s}\right)\left(\begin{array}{cc}
M & 0 \\
0 & 0
\end{array}\right)=\left(\hat{w}_{n-s+1}, 0_{s-1}\right)
$$

where $\hat{w}_{n-s+1}$ has $n-s+1$ elements 


$$
d_{s+i+1, i+1}-d_{s+i, i},(i=0,1, \cdots, n-s+1),
$$

and $d_{s, 0}=d_{n+1, n-s+1}=0$. This is $V\left[A, D_{-s}\right]=V_{-s+1}$. Similarly,

$$
V_{-s+2}=V_{-s+1} U_{s-1}(I-A)=V_{-s} U_{s}(I-A) U_{s-1}(I-A) \text {. }
$$

But by Lemma 2, $U_{s}(I-A) U_{s-1}=U_{s}(I-A)$. Thus

$$
V_{-s+2}=V_{-s} U_{s}(I-A)^{2},
$$

and by induction it follows that if $k \leqq s$,

$$
V_{-s+k}=V_{-s} U_{s}(I-A)^{k} \text {. }
$$

In particular,

$$
V_{-s+s}=V_{-s} U_{s}(I-A)^{s} \text {. }
$$

Case (iii). When $k>s$, we divide the problem into two parts. Using case (i) we have

$$
V_{-s+k}=V_{-s+s}(I-A)^{k-s} L_{k-s} .
$$

But by case (ii), $V_{-s+s}=V_{-s} U_{s}(I-A)^{s}$. Thus

$$
\begin{aligned}
V_{-s+k} & =V_{-s} U_{s}(I-A)^{s}(I-A)^{k-s} L_{k-s} \\
& =V_{-s} U_{s}(I-A)^{k} L_{k-s} .
\end{aligned}
$$

This completes the proof of the lemma.

Using the above lemmas we prove Theorem 1, which establishes a precise upper bound for $k$ in the case where $A$ is nilpotent and $[A, X]_{k}=P(A) \neq 0$.

THEOREM 1. Let $A$ be an $n \times n$ nilpotent nonderogatory matrix. Let $p$ be a positive integer such that $p<n$. Let

$$
\lambda_{i}(i=p, p+1, \cdots, n-1)
$$

be scalars from $F$ such that $\lambda_{p} \neq 0$. Then there exists a matrix $X$ such that

$$
[A, X]_{k}=\sum_{i=p}^{n-1} \lambda_{i} A^{i} \neq 0
$$

if and only if $k \leqq 2 p$.

Proof. We first prove the case where $\lambda_{i}=0$ for all $i>p$. We may assume without loss of generality that $\lambda_{p}=1$ since $[A, X]_{k}=$ $A^{p}$ if and only if $\left[A, \lambda_{p} X\right]_{k}=\lambda_{p} A^{p}$. 
If there exists a matrix $X$ satisfying (16) where $A$ is nilpotent, then $[A, X]_{k}=\left[A, \sum_{s=-n+1}^{n-1} D_{s}\right]_{k}=A^{p}$. Thus we must have

$$
\left[A, D_{s-l k}\right]_{k}=\left\{\begin{array}{l}
0 \quad \text { if } s \neq p \\
A^{p} \text { if } s=p
\end{array} .\right.
$$

Therefore, for $s=p$,

$$
\begin{aligned}
V\left[A, D_{p-k}\right]_{k} & =V_{(p-k)+k}=V D_{p}=V A^{p} \\
& =(0,0, \cdots, 0,1,1, \cdots, 1),
\end{aligned}
$$

which we will call $\left(0_{p}, E_{n-p}\right)$. If $k \leqq p$, from (11),

$$
V_{(p-k)+k}=V_{p-k}(I-A)^{k} L_{p} \text {. }
$$

Using an argument similar to that used in proving lemma 2, we find that $(I-A)^{k} L_{p}$ can be written as $\left(\begin{array}{cc}0 & 0 \\ 0 & N_{k}\end{array}\right)$ where $N_{k}$ has dimensions $(n-p+k) \times(n-p)$. Since this matrix has a square submatrix of order $n-p$ with 1's on the diagonal, zeros below, it has rank $n-p$.

Now rewriting (12) as

$$
\left(0_{p}, E_{n-p}\right)=\left(0_{p-k}, w_{n-p+k}\right)\left(\begin{array}{cc}
0 & 0 \\
0 & N_{k}
\end{array}\right)
$$

we see that solving this equation is equivalent to solving $E_{n-p}=$ $\left(w_{n-p+k}\right) N_{k}$. The augmented matrix for this equation is $\left(\begin{array}{l}N_{k} \\ E_{n-p}\end{array}\right)$, and since $N_{k}$ has rank $n-p$, the augmented matrix also has rank $n-p$. Thus the system has a solution with $(n-p+k)-(n-p)=k$ parameters.

Now if $k>p$ we refer to equation (15) and set

$$
V_{(p-k)+k}=V_{p-k} U_{k-p}(I-A)^{k} L_{p} \text {. }
$$

But the product on the right may be written as $\left(\begin{array}{cc}0 & H_{k} \\ 0 & 0\end{array}\right)$.

If $k=2 p$ then $H_{k}$ is square of order $n-p$. Since it has minus signs in a checkerboard pattern, we may transform it into a matrix with nonnegative elements or nonpositive elements (depending on whether $p$ is even or odd) by multiplying on the left and right by the matrix $D=$ diag. $\left(-1,1,-1, \cdots,(-1)^{n-p}\right)$. Thus the determinant of $H_{k}$ will be unchanged and the resulting matrix has determinant

$$
(-1)^{p} \prod_{i=0}^{n-p-1} \frac{\left(\begin{array}{c}
2 p+i \\
p
\end{array}\right)}{\left(\begin{array}{c}
p+i \\
p
\end{array}\right)} \neq 0
$$

(see Muir, Vol. 3, p. 451). Hence $H_{k}$ is nonsingular. Furthermore, 
$(-1)^{p} H_{k}$ is positive definite since the principal subdeterminants are all positive by the same argument.

Thus if $k=2 p$ we may rewrite the equation (18) as

$$
\left(0_{p}, E_{n-p}\right)=\left(\hat{w}_{n-p}, 0_{p}\right)\left(\begin{array}{cc}
0 & H_{k} \\
0 & 0
\end{array}\right) .
$$

But solving this system is equivalent to solving

$$
E_{n-p}=\widehat{w}_{n-p} H_{k},
$$

and since $H_{k}$ is nonsingular, this system has a unique solution. A solution for $k=2 p$ implies the existence of matrices $X$ satisfying $[A, X]_{k}=A^{p}$ for all $k<2 p$.

Next we show that there is no solution for $k=2 p+1$, and thus for any $k>2 p$, by the following argument. Since $H_{k}$ is nonsingular, equation (19) is equivalent to $E_{n-p} H_{k}^{-1}=\widehat{w}_{n-p}$. Multiplying both sides of this equation by the $(n-p) \times 1$ column vector $E_{n-p}^{T}$ gives

$$
E_{n-p} H_{k}^{-1} E_{n-p}^{T}=\widehat{w}_{n-p} E_{n-p}^{T}=\sum_{i=1}^{n-p} d_{p+i, i} .
$$

This is the sum of the nonzero elements in $D_{-p}$. By Lemma 1, if $[A, X]=D_{-p}$, then $\sum_{i=1}^{n-p} d_{p+i, i}=0$. But since $(-1)^{p} H_{k}$ is positive definite, $(-1)^{p} H_{k}^{-1}$ is also. Thus the product on the left in (20) is not zero and there does not exist a solution for $k>2 p$.

This completes the proof in the case where $[A, X]_{k}=\lambda A^{p}$. In the case where $[A, X]_{k}=\lambda_{p} A^{p}+\lambda_{p+1} A^{p+1}+\cdots+\lambda_{n-1} A^{n-1}$, we see that $X$ may be written as $\sum_{i=p}^{n-1} X_{1 i}$ where $\left[A, X_{1 i}\right]_{k}=\lambda_{i} A^{i}$.

If $A$ is derogatory then the Jordan canonical form for $A$ is diag. $\left(A_{1}, A_{2}, \cdots, A_{s}\right)$ where $s>1$. Theorem 1 can also be extended to the derogatory case. The method of proof is similar to that used in Theorem 1.

THeORem 2. Let $A$ be an $n \times n$ nilpotent matrix. Let $p$ be a positive integer such that $p<n_{i}$ where $n_{i}$ is the dimension of the largest block in the Jordan canonical form for $A$. Let $\lambda_{i}(i=p$, $p+1, \cdots, n-1)$ be scalars from $F$ such that $\lambda_{p} \neq 0$. Then there exists a matrix $X$ such that

$$
[A, X]_{k}=\sum_{i=p}^{n_{i}-1} \lambda_{i} A^{i} \neq 0
$$

if and only if $k \leqq 2 p$.

Some remarks about the integer $p$ are in order here. If the Jordan canonical form for $A$ is $\operatorname{diag}$. $\left(A_{1}, A_{2}, \cdots, A_{s}\right)$ we may assume without 
loss of generality that the dimension $n_{i}$ of $A_{i}$ is greater than or equal to the dimension $n_{i+1}$ of $A_{i+1}$ for $i=1,2, \cdots, s-1$. Since $A^{p}=$ diag. $\left(A_{1}^{p}, A_{2}^{p}, \cdots, A_{s}^{p}\right), p$ must be less than $n_{1}$ if $A^{p}$ is to be different from zero. However, $A_{i}^{p}$ may be zero for some $i>1$.

Notice that since the Jordan canonical form for a nilpotent matrix is the same as the rational canonical form for that matrix, the constructions for the matrices $X$ in Theorems 1 and 2 may be done with rational operations.

The general case. Here it is not assumed that $A$ is nilpotent. We assume that $A$ is in Jordan canonical form. Again we choose a polynomial $P(A)$ which we desire to write as a higher commutator of $A$. Theorems 3 and 4 establish the maximal value for $k$ in equation (4).

TheOREM 3. Let $A$ be an $n \times n$ nonderogatory matrix in Jordan canonial form $\alpha I+N$ where $N$ is the nilpotent matrix with 1's on the first superdiagonal and zeros elsewhere. Let $P(A)$ be a polynomial in $A$ such that $P(A) \neq 0$. Let $t$ be the multiplicity of $\alpha$ as a root of $P(x)$. Then there exists an $n \times n$ matrix $X$ such that

$$
[A, X]_{k}=P(A)
$$

if and only if $k \leqq 2 t$.

$$
\begin{aligned}
& \text { Proof. If } A=(\alpha I+N) \text { then } \\
& \qquad[A, X]_{k}=[(\alpha I+N), X]_{k}=[\alpha I, X]_{k}+[N, X]_{k}=[N, X]_{k} .
\end{aligned}
$$

Thus condition (24) becomes $[N, X]_{k}=P(\alpha I+N)=\sum_{i=1}^{n-1} \lambda_{i} N^{i}$ where $\lambda_{i}=p^{(i)}(\alpha) / i$. Now by Theorem 1 , (24) has a solution if and only if $k \leqq 2 t$.

Theorem 4. Let $A=$ diag. $\left(A_{1}, A_{2}, \cdots, A_{s}\right)$ where $A_{i}=\left(\alpha_{i} I+N_{i}\right)$ $(i=1,2, \cdots, s)$ where each $N_{i}$ is as in Theorem 3. Let $P$ be a polynomial such that $P(A) \neq 0$. Let $A_{i_{1}}, A_{i_{2}}, \cdots, A_{i_{t}}$ be the blocks of $A$ such that $P\left(A_{i_{j}}\right) \neq 0$. Let $m_{i_{j}}$ be the multiplicity of $\left(x-\alpha_{i_{j}}\right)$ in $P(x)$. Let $m=\min .\left\{m_{i_{j}}\right\}$. Then there exists an $n \times n$ matrix $X$ such that

$$
[A, X]_{k}=P(A)
$$

if and only if $k \leqq 2 m$.

Proof. If $A=$ diag. $\left(A_{1}, A_{2}, \cdots, A_{s}\right)$ then

$$
P(A)=\text { diag. }\left(P\left(A_{1}\right), P\left(A_{2}\right), \cdots, P\left(A_{s}\right)\right) \text {. }
$$

If $P\left(A_{t}\right)=0$ for some $A_{t}$, then there exists a matrix $X_{t} \neq 0$ such that 
$\left[A_{t}, X_{t}\right]_{k}=P\left(A_{t}\right)=0$ for any positive integer $k$. Thus we need only consider those $A_{i}$ for which $P\left(A_{i}\right) \neq 0$. Assume that $P\left(A_{i}\right) \neq 0$ for all $i=1,2, \cdots, s$. Then if we let

$$
X=\text { diag. }\left(X_{1}, X_{2}, \cdots, X_{s}\right)
$$

where $\left[A_{i}, X_{i}\right]_{k}=P\left(A_{i}\right)$, the matrix $X$ will satisfy (25). Assume without loss of generality that the degree of $\left(x-\alpha_{1}\right)$ in $P(x)$ is $m=$ min. $\left\{m_{i}\right\}$. Then $\left[A_{1}, X_{1}\right]=P\left(A_{1}\right)$ if and only if $k \leqq 2 m$. Thus $[A, X]_{k}=$ $P(A)$ if and only if $k \leqq 2 m$.

\section{BIBLIOGRAPHY}

1. M.A. Drazin, J.W. Dungey, and K.W. Gruenberg, Some theorems on commutative matrices, J. London Math. Soc. 26 (1951) 221-228.

2. N. Jacobson, Lie Algebras, Interscience Publishers, New York, 1962.

3. N. H. McCoy, On quasi-commutative matrices, Trans. Amer. Math. Soc. 36 (1934), $327-340$.

4. N. T. Muir, Theory of determinants, Dover, New York, 1920.

5. W. V. Parker, Matrices and polynomials, Math. Monthly 61 (1954), 182-183.

6. - The matrix equation $A X=X B$, Duke Math. J. 17 (1950), 43-51.

7. W. V. Parker and J. C. Eaves, Matrices, Ronald Press, New York, 1960.

8. W.E. Roth, On K-commutative matrices, Trans. Amer. Math. Soc. 39 (1936), 483-495.

9. O. Taussky, and H. Wielandt, Linear relations between higher additive commutators, Proc. Amer. Math. Soc. 13 (1962), 732-735.

Received December 6, 1968.

Tennessee Technological University 



\section{PACIFIC JOURNAL OF MATHEMATICS}

\section{EDITORS}

\author{
H. SAMELSON \\ Stanford University \\ Stanford, California 94305 \\ Richard Pierce \\ University of Washington \\ Seattle, Washington 98105
}

J. DUGUNDJI

Department of Mathematics

University of Southern California

Los Angeles, California 90007

BASIL GORDON*

University of California

Los Angeles, California 90024

\section{ASSOCIATE EDITORS}
E. F. BECKENBACH
B. H. NeUmanN
F. WOLF
K. YoSHIDA

\section{SUPPORTING INSTITUTIONS}

UNIVERSITY OF BRITISH COLUMBIA CALIFORNIA INSTITUTE OF TECHNOLOGY

UNIVERSITY OF CALIFORNIA MONTANA STATE UNIVERSITY

UNIVERSITY OF NEVADA

NEW MEXICO STATE UNIVERSITY

OREGON STATE UNIVERSITY

UNIVERSITY OF OREGON

OSAKA UNIVERSITY

UNIVERSITY OF SOUTHERN CALIFORNIA
STANFORD UNIVERSITY

UNIVERSITY OF TOKYO

UNIVERSITY OF UTAH

WASHINGTON STATE UNIVERSITY

UNIVERSITY OF WASHINGTON

AMERICAN MATHEMATICAL SOCIETY CHEVRON RESEARCH CORPORATION TRW SYSTEMS

NAVAL WEAPONS CENTER 


\section{Pacific Journal of Mathematics}

\section{Vol. 32, No. $1 \quad$ January, 1970}

Robert Alexander Adams, Compact Sobolev imbeddings for unbounded domains ........................................ 1

Bernhard Amberg, Groups with maximum conditions .................. 9

Tom M. (Mike) Apostol, Möbius functions of order k............... 21

Stefan Bergman, On an initial value problem in the theory of two-dimensional transonic flow patterns ................... 29

Geoffrey David Downs Creede, Concerning semi-stratifiable spaces ...... 47

Edmond Dale Dixon, Matric polynomials which are higher

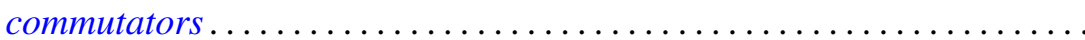

R. L. Duncan, Some continuity properties of the Schnirelmann density.

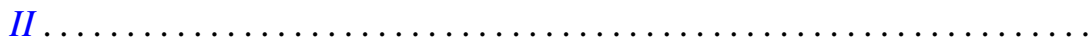

Peter Larkin Duren and Allen Lowell Shields, Coefficient multipliers of $H^{p}$

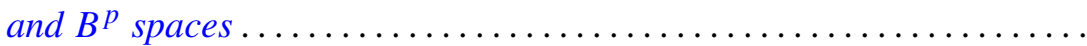

Hector O. Fattorini, On a class of differential equations for vector-valued

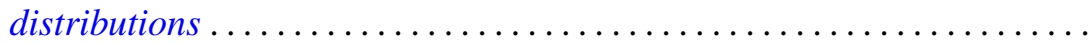

Charles Hallahan, Stability theorems for Lie algebras of derivations. . . . . . 105

Heinz Helfenstein, Local isometries of flat tori ................ 113

Gerald J. Janusz, Some remarks on Clifford's theorem and the Schur

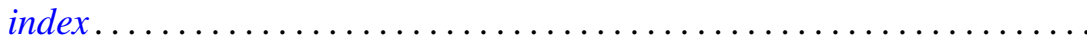

Joe W. Jenkins, Symmetry and nonsymmetry in the group algebras of discrete groups. ...

Herbert Frederick Kreimer, Jr., Outer Galois theory for separable

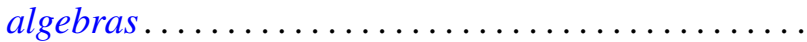

D. G. Larman and P. Mani, On visual hulls

R. Robert Laxton, On groups of linear recurrences. II. Elements of finite order.

Dong Hoon Lee, The adjoint group of Lie groups ...

James B. Lucke, Commutativity in locally compact rings

Charles Harris Scanlon, Rings of functions with certain Lipschitz

$$
\text { properties ............................... }
$$

Binyamin Schwarz, Totally positive differential systems .

James McLean Sloss, The bending of space curves into piecewise helical curves.

James D. Stafney, Analytic interpolation of certain multiplier spaces ...

Patrick Noble Stewart, Semi-simple radical classes.......

Hiroyuki Tachikawa, On left $\mathrm{QF}-3$ rings ...................... 255

Glenn Francis Webb, Product integral representation of time dependent nonlinear evolution equations in Banach spaces.... . . . 